\title{
Is a good perioperative echocardiographic result predictive of durability in ischemic mitral valve repair?
}

K. Serri, MD, D. Bouchard, MD, P. Demers, MD, M. Coutu, MD, M. Pellerin, MD, M. Carrier, MD, L. P. Perrault, MD, PhD, R. Cartier, MD, P. Pagé, MD, M. Cossette, MSc, and A. J. Basmadjian, MD, MSc

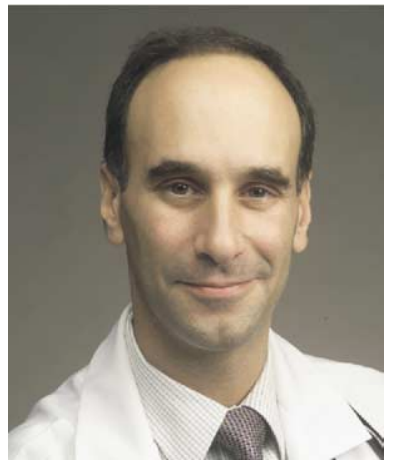

Dr. Basmadjian

Supplemental material is available online.
From the Montreal Heart Institute, University of Montreal, Montreal, Quebec, Canada.

Read at the Eighty-fifth Annual Meeting of The American Association for Thoracic Surgery, San Francisco, Calif, April 10-13, 2005.

Received for publication July 7, 2005; revisions received Oct 10, 2005; accepted for publication Oct 20, 2005.

Address for reprints: A. J. Basmadjian, MD, MSc, Montreal Heart Institute, Research Center, 5000 Belanger, Montreal, Quebec, Canada, H1T 1C8 (E-mail: arsene.basmadjian@icm-mhi.org).

J Thorac Cardiovasc Surg 2006;131:565-73

$0022-5223 / \$ 32.00$

Copyright (C) 2006 by The American Association for Thoracic Surgery

doi:10.1016/j.jtcvs.2005.09.037
Background: Chronic ischemic mitral regurgitation is associated with poor longterm survival. Despite the increasing popularity of valve repair, its durability and long-term outcome for ischemic mitral regurgitation have recently been questioned.

Methods: Seventy-eight patients underwent repair for ischemic mitral regurgitation between 1996 and 2002 at our institution. Of these patients, 73 had complete clinical and echocardiographic follow-up. Preoperative, intraoperative, and postoperative clinical data were obtained, and the results of echocardiograms were reviewed to assess the rate of recurrence of regurgitation after repair and to identify predictive factors.

Results: The mean preoperative mitral regurgitation grade, New York Heart Association class, and left ventricular ejection fraction were 2.72, 2.65, and 39.4\%, respectively. Mortality was $12.3 \%$ at 30 days and $30.1 \%$ at a mean follow-up of 39 \pm 25 months. Immediate postoperative echocardiography showed absent or mild mitral regurgitation in $89.4 \%$ of patients and showed moderate mitral regurgitation in $10.6 \%$. Freedom from reoperation was $93.2 \%$. Recurrent moderate mitral regurgitation $(2+)$ was present in $36.7 \%$ of patients, and severe mitral regurgitation (3+ to $4+$ ) was present in $20.0 \%$ at mean follow-up of $28.1 \pm 22.5$ months. Only age $(P=.0130)$ and less marked preoperative posterior tethering $(P=.0362)$ were predictive of recurrent mitral regurgitation. Patients with a preoperative New York Heart Association class greater than II and recurrent mitral regurgitation greater than $2+$ had decreased survival $(P=.0152$ and $P=.0450$, respectively).

Conclusions: Significant recurrent mitral regurgitation occurs following repair for ischemic mitral regurgitation, despite good early results. This finding raises questions about the need for improved repair techniques, better patient selection, or eventual mitral valve replacement in selected patients.

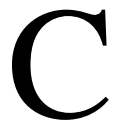
hronic ischemic mitral regurgitation (IMR) is generally defined as mitral regurgitation (MR) secondary to myocardial infarction. ${ }^{1}$ IMR has been shown to be an independent predictor of mortality, ${ }^{2-4}$ with reported survival of $40 \%$ to $60 \%$ at 5 years. ${ }^{1,5-8}$ Operative mortality is increased for patients undergoing mitral valve operation and revascularization in the presence of IMR when compared with nonischemic $\mathrm{MR}^{4,6,7}$ or revascularization alone.

Surgical correction, either by replacement or repair, is generally recommended for $3+$ and $4+$ IMR. $^{9-11}$ In comparative analyses, repair seems to benefit most patients with IMR, with the possible exception of high-risk groups (higher New York Heart Association [NYHA] functional class or emergency operation). ${ }^{1,12}$ In patients who undergo repair, residual MR greater than $1+$ is associated with increased late mortality. ${ }^{13}$

Mitral valve repair, which most often involves the use of an undersized annuloplasty ring, has been shown to be effective, with $98 \%$ short-term success. ${ }^{1}$ Data 

Abbreviations and Acronyms
AVR = aortic valve replacement
IMR = ischemic mitral regurgitation
$\mathrm{LV}=$ left ventricle
LVEF $=$ left ventricular ejection fraction
MR = mitral regurgitation
NYHA $=$ New York Heart Association

concerning MR recurrence after repair in patients with degenerative mitral valve disease have recently been published and show a significant incidence of late MR. ${ }^{14}$ Longterm results of repair for IMR are not defined, in part because of underreporting of failure rates and because death is an important competing end point in the evaluation of repair failure. Although good results have been reported, ${ }^{15}$ high recurrence rates of MR at follow-up have recently been published, and this raises questions about the long-term results of mitral valve repair. ${ }^{5,16}$ Moreover, very few studies have evaluated predictors of recurrence.

Therefore, our objective was to analyze the incidence of MR recurrence after mitral valve repair for IMR and attempt to define possible predictors of recurrence by looking at preoperative, perioperative, and postoperative clinical and echocardiographic data.

\section{Patients and Methods}

All patients who had mitral valve repair performed at the Montreal Heart Institute between 1996 and 2002 were considered. Seventyeight patients with MR resulting from myocardial infarction ${ }^{1}$ with normal mitral valve leaflets and chordae were identified. Of these patients, 73 (94\%) had long-term postoperative clinical and echocardiographic follow-up and were included in the study.

\section{Patient Characteristics}

The following patient data were analyzed: age; comorbidities (including hypertension, diabetes, and preoperative atrial fibrillation); extent of coronary artery disease on preoperative coronary angiogram (significant lesions were defined as $>50 \%$ narrowing of a major coronary artery); previous myocardial infarction, either recent (within 30 days before surgery) or old; left ventricular ejection fraction (LVEF) and NYHA functional class before surgery and at last follow-up; repeat operation; associated valvular lesions; urgent operation (defined as within 48 hours of admission); and preoperative intra-aortic balloon pump use. Recent myocardial infarctions were defined as a chest pain syndrome associated with either ST segment elevation on the electrocardiogram or cardiac enzymes above the upper limit of normal. A prior myocardial infarction was assumed if an akinetic segment was observed on the echocardiogram, with or without associated Q waves on the electrocardiogram.

\section{Surgical Techniques}

For intraoperative data, we analyzed bypass time, aortic crossclamp time, type of repair, annular size, associated procedures, number of grafts, adequacy of revascularization, postoperative myocardial infarction (defined as creatine kinase MB $>100 \mathrm{U} / \mathrm{L}$ ), and early postoperative death (within 30 days or during the index hospitalization). Revascularization was deemed complete only if all coronary arteries with significant lesions ( $>50 \%$ stenosis) were grafted, independent of artery caliber or segmental wall motion at baseline.

\section{Echocardiographic Results}

The results of immediate postoperative transesophageal or transthoracic echocardiograms within the first postoperative week were assessed for the presence and severity of early residual MR. After a mitral valve repair procedure at our institution, an echocardiographic examination is performed before discharge, at 6 months, and then every 1 to 2 years. The severity of MR is graded on a scale of 1 to 4 according to color jet area, pulsed wave-Doppler of the pulmonary veins, and proximal isovelocity surface area according to American Society of Electrocardiography guidelines. ${ }^{17}$ The results of preoperative and postoperative echocardiograms were obtained for the severity of MR (graded according to color jet area and pulsed wave-Doppler of the pulmonary veins), LVEF, regional left ventricular (LV) function, LV end-diastolic and endsystolic dimensions, left atrial diameter, systolic pulmonary artery pressure, and associated valvular lesions.

A subgroup of patients had both preoperative and postoperative echocardiograms available for detailed analysis. Echocardiograms were reviewed by 1 observer for the severity of MR, ${ }^{17}$ as well as for the direction of the regurgitant jet (central vs eccentric), LVEF, regional LV wall motion, LV end-diastolic and end-systolic dimensions, left atrial dimensions, left atrial volume, ${ }^{18}$ the presence of associated valvular lesions, and systolic pulmonary artery pressure, as determined from the tricuspid regurgitant jet. The LV sphericity index was measured by calculating the ratio between the LV major axis and minor axis dimensions in the apical 4-chamber view. ${ }^{19-21}$ As this ratio decreases and approaches unity, the LV becomes more spherical. The mitral annular diameter was measured in the apical 4- and 2-chamber views, and the mean annular diameter was calculated. The degree of anterior and posterior tethering was estimated by measuring the distance between the anterior mitral annulus and the anterior or posterior papillary muscle tip, respectively (Figure E1). Tenting height and area were also measured. ${ }^{22}$

\section{Clinical Follow-up}

Follow-up was performed at a specialized valvular heart disease outpatient clinic. Most patients were seen for follow-up, whereas a few patients were followed up through mail correspondence.

\section{Statistical Analysis}

Patient characteristics and echocardiographic results are expressed as means $\pm \mathrm{SD}$ or simple frequencies and percentages. Late mortality was studied by using survival analysis. Survival curves were computed by using the Kaplan-Meier formulas and were compared between groups by using the log-rank test. Univariate and multivariate linear regression were used to identify predictors of MR recurrence. To detect any differences between the echocardiographic subgroup and the rest of the study population, independent sample $t$ tests were performed on normally 
TABLE 1. Baseline patient characteristics $(n=73)$

\begin{tabular}{lc}
\hline Variable & Data \\
\hline Age (y) & $65 \pm 9.9$ \\
Male & $49(67)$ \\
HBP & $38(52)$ \\
Diabetes & $24(33)$ \\
Atrial fibrillation & $26(36)$ \\
LVEF (\%) & $39.4 \pm 12.2$ \\
NYHA class & $2.65 \pm 0.81$ \\
0 & $0 \%$ \\
I & $913 \%$ \\
II & $2534 \%$ \\
$\quad$ III-IV & $3953 \%$ \\
LV diastolic diameter (mm) & $58.2 \pm 6.5$ \\
LV systolic diameter (mm) & $44.3 \pm 8.0$ \\
LA diameter (mm) & $45.5 \pm 6.5$ \\
PAP (mm Hg) & $45.8 \pm 16.2$ \\
Preoperative MR grade & $2.72 \pm 0.51$ \\
CAD & \\
$\quad$ One vessel & $8(11)$ \\
$\quad$ Two vessels & $13(18)$ \\
$\quad$ Three vessels & $48(66)$ \\
Recent MI & $21(29)$ \\
Previous MI & $62(85)$ \\
\hline
\end{tabular}

Data are $\mathrm{n}(\%)$ or mean $\pm \mathrm{SD}$. HBP, High blood pressure; $L V E F$, left ventricular ejection fraction; $N Y H A$, New York Heart Association; $L V$, left ventricular; $L A$, left atrial; $P A P$, pulmonary artery pressure; $M R$, mitral regurgitation; $C A D$, coronary artery disease; $M I$, myocardial infarction.

distributed continuous variables, and the nonparametric MannWhitney test was used when distributional assumptions were not met. Paired-samples $t$ tests were used to compare continuous variables from the echocardiographic subgroup. Statistical analysis was performed with the computer software SAS (SAS Institute Inc, Cary, NC).

\section{Results}

\section{Patient Characteristics}

The baseline characteristics of the 73 patients are shown in Table 1. The mean age was 65 years, the mean ejection fraction was $39.4 \% \pm 12.2 \%$, and the average NYHA functional class was $2.65 \pm 0.81$. Patients had a dilated LV and left atrium. The mean preoperative MR grade was 2.72; moderate MR $(2+)$ was present in $37 \%$ of patients and severe MR $(3+$ and $4+)$ in $63 \%$ (Figure 1$)$. The operative characteristics of the patients are shown in Table 2. Coronary artery bypass grafting was performed in $87.7 \%$ of patients at the time of mitral valve repair, and among the 9 remaining patients, 5 had already undergone coronary artery bypass grafting during a prior procedure. The other 4 did not require coronary artery bypass grafting because of either patent or chronically occluded vessels.

Repair involved insertion of an undersized annuloplasty ring in all patients; 2 patients also had an Alfieri stitch procedure performed. The degree of undersizing was not
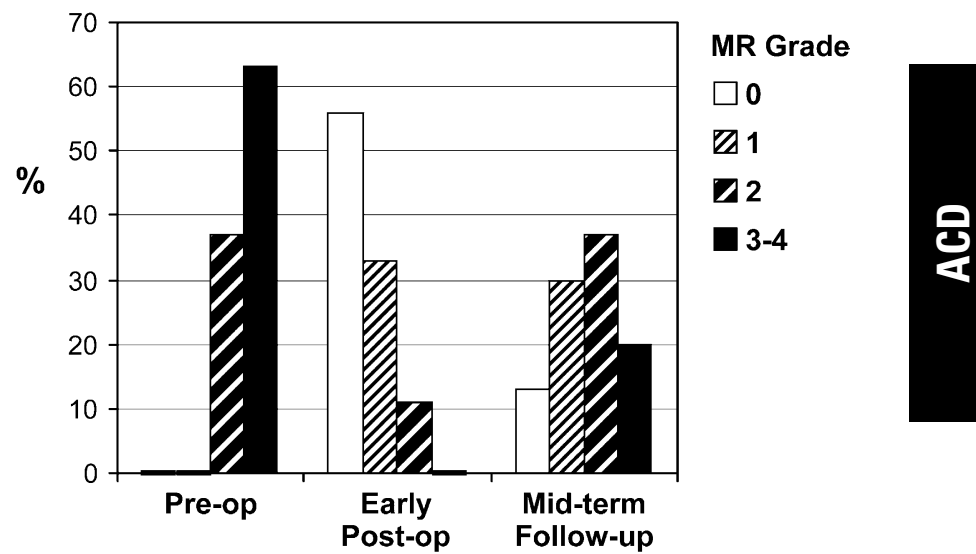

Figure 1. Preoperative, early postoperative (within 30 days), and mid-term (28 \pm 22 months) follow-up MR grade.

homogenously recorded in the 6-year period of the study. Men and large patients tended to receive a size 28 ring, whereas women or smaller patients received a size 26 ring. Specifically, a size 26 ring was used in $39 \%$, size 28 in $44 \%$, size 30 in $8 \%$, size 32 in 7\%, and size 34 in $2 \%$ of patients. Ninety percent of patients had a rigid or semirigid complete ring (79\% Carpentier Physio rings, 2\% Carpentier Classic rings, Edwards Lifesciences, LLC, Irvine, Calif. 7\% Annuloflo rings, Carbomedics, Sorin Group Company, Austin, Tex. and 2\% Seguin rings, Edwards Lifesciences, LLC, Irvine, Calif.). A Cosgrove flexible posterior band was used in $10 \%$ of patients. Repair was deemed successful in the operating room if MR was equal to or less than 1.

Additional interventions were performed in a few patients: 6 patients had tricuspid annuloplasty for grade $3 / 4$ or greater regurgitation, 3 patients had LV operations (aneurysm resection or a remodeling procedure), and 2 patients had aortic valve replacement (AVR). The outcome of patients who underwent LV operation or AVR was good, with no early deaths and with mild MR at late follow-up. Patients

TABLE 2. Operative characteristics $(n=73)$

\begin{tabular}{lc}
\hline Variable & Data \\
\hline Reoperation & $14(19)$ \\
Urgent operation & $6(8)$ \\
Preoperative IABP & $8(11)$ \\
Bypass time (min) & $127.5 \pm 36.5$ \\
Crossclamp time (min) & $88.5 \pm 28.0$ \\
Number of grafts & $2.2 \pm 1.2$ \\
Incomplete revascularization & $26(37)$ \\
Annular size (mm) & $27.6 \pm 1.8$ \\
Postoperative MI & $12(16)$
\end{tabular}

Data are $\mathrm{n}(\%)$ or mean $\pm \mathrm{SD}$. IABP, Intra-aortic balloon pump; $M I$, myocardial infarction. 
who had tricuspid annuloplasty had a worse outcome during follow-up: 2 died within 30 days after the operation, and 1 died later. One patient required early mitral valve replacement because of severe MR, and the remaining 2 patients had moderate MR at last follow-up.

\section{Survival, Reoperation, and Clinical Follow-up}

The early postoperative death rate, defined as death within 30 days after surgery or within the same hospitalization, was $12.3 \%$. The mean survival follow-up was $39.3 \pm 25.0$ months. Actuarial survival was $85 \%$ at 1 year, $79 \%$ at 2 years, and $68 \%$ at 5 years (Figure E2). Actuarial freedom from reoperation was $93.2 \%$. At follow-up, the mean NYHA class was significantly improved, at $1.56 \pm 0.63$ $(P<.0001)$; LVEF was not significantly changed, at $38.9 \%$ $\pm 12.9 \%$. Neither was associated with the MR grade at follow-up.

\section{Recurrence of MR}

Results of an immediate postoperative transesophageal echocardiogram or a transthoracic examination performed within the first postoperative week were available in $90 \%$ of the patients. Mild or absent MR ( 0 or $1+)$ was observed in $89.4 \%$ of patients, whereas moderate MR $(2+)$ was noted in $10.6 \%$ (7 patients). No patient had severe MR $(3+$ or $4+)$ on early postoperative echocardiogram (Figure 1). The evolution of the 7 cases with early moderate MR was poor: 2 patients died within 3 months after surgery, 2 patients required mitral valve replacement, 2 patients had $2+$ MR at last follow-up, and 1 patient improved to $1+\mathrm{MR}$.

Mid-term echocardiographic follow-up $(28.1 \pm 22.5$ months) results were available in all 60 patients who survived the early postoperative period. At the last examination available, moderate MR $(2+)$ was observed in $37 \%$ of our patients, and severe MR $(3+$ or $4+)$ was observed in $20 \%$ (Figures 1 and E3). Recurrence of severe MR occurred both early and late, within 6 months after surgery in 5 patients and after 6 months in 7 patients. Five patients (8.3\%) required reoperation, and all 5 received mitral valve replacement ( 3 mechanical prostheses and 2 bioprostheses). The interval between initial operation and reoperation varied from 14 days to 38.5 months. Recurrent severe MR developed in the remaining 7 patients in a period ranging from 3 months to 62 months after surgery, but they had not undergone reoperation at last follow-up.

\section{Detailed Echocardiographic Analysis}

Echocardiographic results for the subgroup of 18 patients with preoperative and postoperative echocardiograms available for detailed analysis are shown in Table 3. MR grade significantly decreased with operation $(P<.0001)$. There was no significant change in LVEF, sphericity index, LV or LA dimensions, or volumes between preoperative and
TABLE 3. Echocardiographic subgroup results $(n=18)$

\begin{tabular}{lccc}
\hline Variable & Before surgery & After surgery & $P$ value \\
\hline MR grade & $2.86 \pm 0.56$ & $1.42 \pm 0.83$ & $<.0001$ \\
Eccentric jet (\%) & 33 & 12 & .05 \\
LVEF (\%) & $34.40 \pm 11.45$ & $34.53 \pm 14.22$ & .75 \\
LV diastole (mm) & $60.86 \pm 6.70$ & $59.53 \pm 7.05$ & .34 \\
LV systole (mm) & $44.83 \pm 7.09$ & $45.00 \pm 9.85$ & .97 \\
EDV (mL) & $147.78 \pm 40.03$ & $131.17 \pm 44.16$ & .25 \\
ESV (mL) & $106.22 \pm 38.28$ & $99.00 \pm 43.47$ & .54 \\
LAD (PSLAX) (mm) & $47.38 \pm 5.93$ & $47.50 \pm 6.29$ & .95 \\
Longitudinal LAD & $62.61 \pm 11.59$ & $59.50 \pm 8.69$ & .37 \\
$\quad$ (A4C) (mm) & & & \\
Transverse LAD & $46.89 \pm 7.83$ & $45.39 \pm 5.99$ & .51 \\
$\quad$ (A4C) (mm) & & & \\
LA volume (mL) & $79.44 \pm 28.42$ & $72.77 \pm 28.05$ & .76 \\
Annular diameter & $32.89 \pm 3.90$ & $26.44 \pm 4.10$ & $<.0001$ \\
$\quad$ (mm) & & & \\
Sphericity index & $1.35 \pm 0.32$ & $1.29 \pm 0.30$ & .42 \\
Anterior tethering & $40.61 \pm 7.57$ & $38.22 \pm 9.83$ & .41 \\
$\quad$ distance (mm) & & & \\
Posterior tethering & $39.39 \pm 8.44$ & $39.22 \pm 6.91$ & .95 \\
$\quad$ distance (mm) & & & \\
Tenting area (cm ${ }^{2}$ ) & $1.48 \pm 0.44$ & $1.09 \pm 0.35$ & .01 \\
Tent height (cm) & $0.78 \pm 0.15$ & $0.68 \pm 0.15$ & .09 \\
Systolic PAP (mm & $46.13 \pm 15.22$ & $40.38 \pm 10.49$ & .72 \\
$\quad$ Hg) & & & \\
\hline Data are mean \pm SD & & &
\end{tabular}

Data are mean \pm SD unless otherwise noted. $M R$, Mitral regurgitation $L V E F$, left ventricular ejection fraction; $L V$, left ventricular; $E D V$, enddiastolic volume; $E S V$, end-systolic volume; $L A D$, left atrial diameter; $L A$, left atrial; $P A P$, pulmonary artery pressure; PSLAX, parasternal long-axis; $A 4 C$, apical 4-chamber.

follow-up echocardiographic studies. There was a significant decrease in annular diameter $(P<.0001)$ and mitral valve tenting area $(P=.0113)$. Mitral anterior and posterior tethering distances were increased before surgery but were not changed at follow-up.

The only preoperative variable that correlated with MR grade at follow-up was the degree of posterior leaflet tethering $(P=.0362)$. A shorter preoperative tethering distance was associated with a greater degree of recurrent MR. At follow-up, none of the postoperative variables correlated with MR severity. We also assessed whether the degree of change of echocardiographic variables was associated with MR grade at follow-up; no statistically significant correlation was observed.

The baseline variables of the echocardiographic subgroup were compared with those of the overall study population to see whether it was a representative subgroup. No significant differences were observed for preoperative MR grade, postoperative MR grade, preoperative NYHA class, postoperative NYHA class, age, or postoperative LVEF $(P>.05)$. Only preoperative LVEF differed between the echocardiographic subgroup and the overall study 
group (34.4\% vs $40.9 \%$, respectively), with borderline statistical significance $(P=.0487)$.

\section{Predictive Factors of Clinical Outcome and MR Recurrence}

In univariate analysis, only age was associated with MR grade at follow-up $(P=.0130)$, and preoperative LVEF showed a borderline association $(P=.06)$. No association was observed for preoperative NYHA functional class, LV dimensions, eccentricity of the regurgitant jet, presence of a recent preoperative or postoperative myocardial infarction, incomplete revascularization, ring type, or annular size. In a multiple linear regression model including age and preoperative LVEF, age remained a statistically significant predictor of MR recurrence but not preoperative LVEF ( $P=$ .0336 and $P=.1695$, respectively; $r^{2}=.1312$ ). MR grade on early postoperative echocardiogram and MR grade at follow-up were not correlated $(P=.1136)$.

Posterior leaflet tethering, being the only echocardiographic variable significantly associated with MR grade at follow-up, a simple linear regression model was used on the echocardiographic subgroup that showed the degree of preoperative posterior leaflet tethering to be a good predictor of late MR recurrence $\left(P=.0362 ; r^{2}=.2463\right)$. Patients with a posterior leaflet tethering distance less than $40 \mathrm{~mm}$ had a greater degree of MR at follow-up (1.65 vs 0.8, respectively; $P=.0459$ ).

The inclusion of timing of the mid-term echocardiographic follow-up as a covariate in the multiple linear regression model did not significantly alter any of these results. Kaplan-Meier survival curves showed that patients with a preoperative NYHA class greater than II and patients with recurrent MR greater than 2+ had significantly higher mortality rates (Figure 2). No association with survival was observed for age, preoperative LVEF, preoperative MR grade, LV dimensions, or recent myocardial infarction.

\section{Discussion}

\section{Clinical Outcome and MR Recurrence}

This study illustrates the high recurrence rate of MR after mitral valve repair for IMR despite the use of a rigid annuloplasty ring and good early postoperative results. Survival was $85 \%$ at 1 year and $68 \%$ at 5 years, consistent with previous studies, ${ }^{1,5,7}$ thus highlighting the negative prognosis associated with IMR. Freedom from reoperation was high (93.2\%), also consistent with the existing literature. ${ }^{1,5}$ However, freedom from reoperation may overestimate the success of a procedure, because many patients with less than optimal results will not undergo reoperation for various reasons, such as advanced age, poor residual LVEF, good functional class despite severe MR, comorbidities, or the patient's desire. Recurrence of MR is a more precise indicator of procedural success but tends to be reported less frequently. The few published studies concerning postrepair
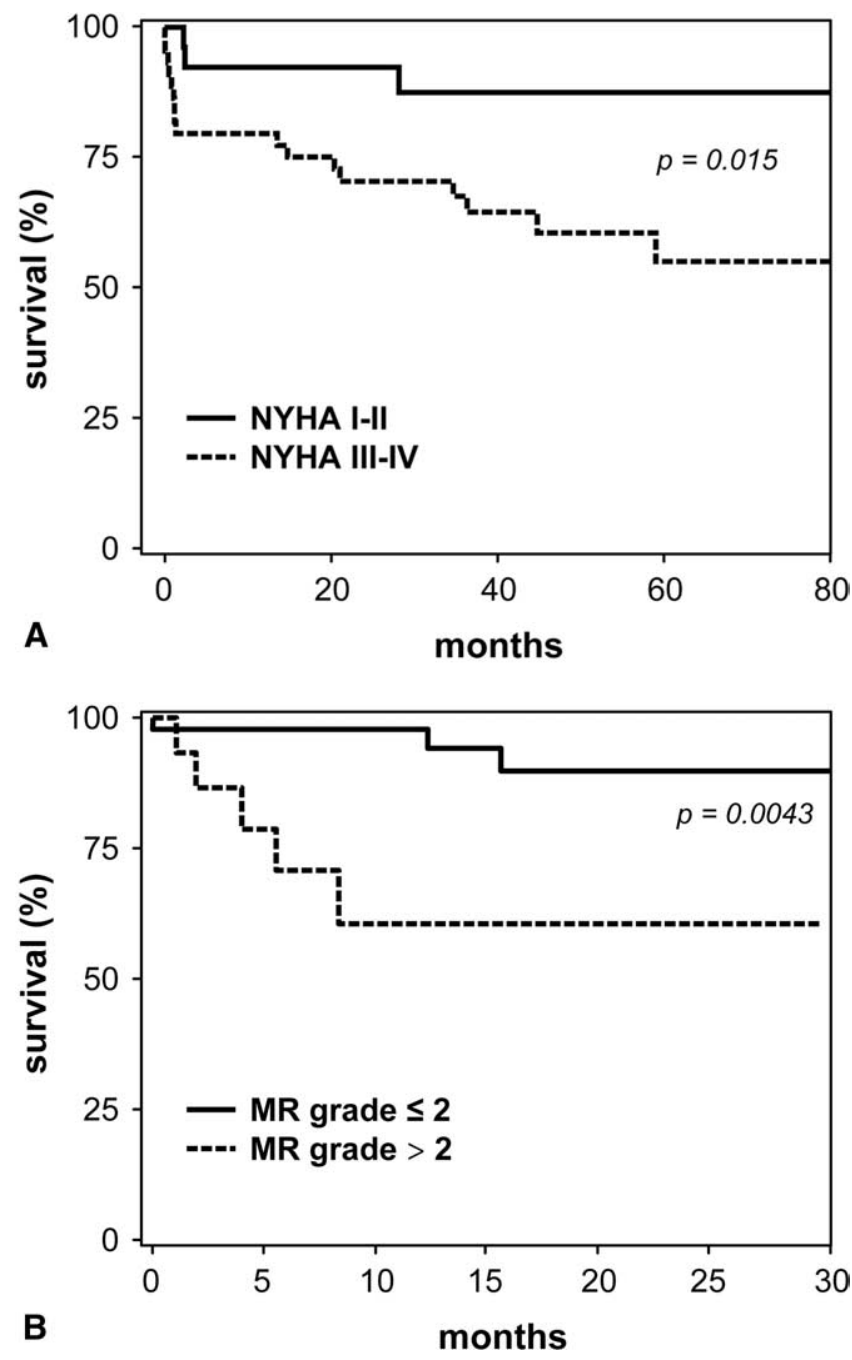

Figure 2. Kaplan-Meier survival curves according to preoperative NYHA class (A) and postoperative MR grade (B). (A) $N=73$ patients. Time " 0 " represents date of surgery. (B) $N=60$ patients having survived to echocardiography. Time " 0 " represents date of latest available echocardiogram.

MR recurrence often have incomplete or short-term echocardiographic follow-up.

Recently, McGee and colleagues ${ }^{5}$ observed that after an annuloplasty procedure for IMR, $28 \%$ of patients had recurrent grade $3+$ or $4+$ MR by 6 months. In our study with complete echocardiographic follow-up, significant rates of recurrent MR were observed both early and late despite very good early postoperative results. This finding is in keeping with those of McGee and colleagues ${ }^{5}$ and of Tahta and associates ${ }^{16}$ but is different from those of Bax and associates, ${ }^{15}$ who reported virtually no MR recurrence at 1.5 years. A possible explanation for this difference is that patients in the Bax study experienced reverse remodeling, 
with significant decreases in LV and left atrial dimensions and improvement in LVEF; this was not shown in our group of patients.

Our study illustrates the importance of long-term follow-up that includes imaging in evaluating the success of any valvular operation. Some patients in our series experienced late severe MR - as long as 5 years after the initial procedurethus emphasizing the need for continued clinical and echocardiographic surveillance.

\section{Predictors of Recurrence}

Among clinical factors, only age was predictive of recurrent MR, with a very weak correlation, however. The degree of LV dysfunction and the severity of preoperative MR did not correlate with the long-term success of the repair procedure. Incomplete revascularization was not correlated with recurrent MR. In the treatment of IMR, ring annuloplasty has been shown to be superior to suture annuloplasty. ${ }^{6,23}$ However, our results, in accordance with previously published studies, ${ }^{5,16}$ do not support an association between annuloplasty ring size and MR recurrence. Although no statistical analysis was performed because of the limited number of patients who had a tricuspid annuloplasty, these patients had a worse clinical outcome than the overall group. This probably reflects a greater disease burden in patients requiring this operation.

On early postoperative echocardiography, $89 \%$ of patients had mild or trivial MR, and no patient had severe MR; this attests to the good early operative results. The MR grade on early postoperative examination was not shown to correlate with the MR grade at follow-up. Only 7 patients had moderate MR on early postoperative echocardiogram, and although there was no statistically significant association with the MR grade at follow-up, these patients seemed to not fare as well as the overall group (among these 7 patients, 2 died early, and 2 required reoperations).

Patients with a preoperative NYHA class greater than II had higher mortality. There was no association between MR severity at follow-up and NYHA functional class. Recurrent MR and survival are both time-dependent and competitive end points, and it is difficult to draw conclusions as to a clear association between them. However, when early deaths were excluded, the survival curves of patients with postoperative MR greater than $2+$ were worse than for those without significant MR recurrence, thus suggesting a possible association between the 2 , as noted by others. ${ }^{24}$

\section{Echocardiographic Predictors of MR Recurrence}

Among echocardiographic factors, the degree of preoperative posterior leaflet tethering correlated with MR grade at follow-up. Leaflet tethering and local LV remodeling have been established as the fundamental mechanisms involved in IMR. ${ }^{25-29}$ It is important to note that patients in our study had increased preoperative mitral leaflet tethering distances. We observed an association between a decreased preoperative posterior papillary muscle tip/anterior annulus distance and a higher MR grade at follow-up; ie, patients with less marked leaflet tethering had more recurrent MR. Previously published work by Kumanohoso and colleagues ${ }^{22}$ showed a greater posterior tethering distance to be independently associated with MR severity. Theirs was a study investigating MR incidence according to infarct location and involved mainly patients with mild MR. One possibility to explain findings in our study is that patients with less severe preoperative tethering may have fewer anomalies to be corrected by reduction annuloplasty than patients with a greater degree of tethering; thus, these patients may be more prone to recurrence. Recent work by Agricola and colleagues ${ }^{30}$ has suggested a new echocardiographic classification of IMR based on tethering pattern, either symmetric or asymmetric, with potential therapeutic implications.

These observations add to the understanding of the complex pathology of IMR, which is more complex than simple annular dilatation; thus, annuloplasty alone may not be the most appropriate treatment. The finding of high postrepair recurrence rates adds incentive to tailor therapy to each patient's pathologic characteristics.

\section{Potential Limitations}

Almost all postoperative echocardiograms were performed at our institution, except for 9 patients who had postoperative studies performed in referring hospitals, usually because of the long traveling distances required. Although this might be a cause for discrepancy for MR in the moderate range, severe MR is exceptionally overreported because our referring centers generally require systolic pulmonary vein flow reversal to report grade $3+$ or $4+$ MR. Echocardiographic data were retrospectively analyzed; however, echocardiograms are prospectively performed in our institution for patients undergoing mitral valve repair, with particular attention directed toward MR recurrence and severity and LV function. Clinical data were prospectively collected. Our study may have been underpowered to detect certain differences because of the limited number of patients, in particular for the echocardiographic subgroup analysis. The subgroup analysis was performed on an unselected number of patients, who were comparable to the overall study population. However, all bias cannot be excluded.

The study does not permit firm recommendations regarding indications or contraindications for IMR repair, repair technique, or valve replacement. However, the results show a high rate of MR recurrence and strongly suggest the need to reevaluate patient selection and repair techniques to improve long-term patient outcome. 


\section{Conclusions}

Significant recurrent MR occurs after mitral valve repair for IMR. Recurrence can occur both early and late, despite good initial surgical results. Postoperative MR was not associated with NYHA class at follow-up but seems to be related to late mortality, thus suggesting the negative prognostic significance of MR recurrence. This raises the question of whether additional repair techniques or better patient selection are needed to improve outcomes. If patients with anticipated repair failure could be identified, these patients could possibly benefit more from mitral valve replacement or other repair techniques. Our findings emphasize the need for long-term prospective studies that include echocardiographic follow-up.

We thank Dr Ihor Dydra for his dedication and guidance, and Mrs Manon Bellemare for her continued devotion to the MHI Valve Clinic.

\section{References}

1. Gillinov AM, Wierup PN, Blackstone EH, et al. Is repair preferable to replacement for ischemic mitral regurgitation? J Thorac Cardiovasc Surg. 2001;122:1125-41.

2. Hickey MS, Smith LR, Muhlbaier LH, et al. Current prognosis of ischemic mitral regurgitation. Implications for future management. Circulation. 1988;78:I51-9.

3. Lamas GA, Mitchell GF, Flaker GC, et al. Clinical significance of mitral regurgitation after acute myocardial infarction. Survival and Ventricular Enlargement Investigators. Circulation. 1997;96:827-33.

4. Cohn LH, Rizzo RJ, Adams DH, et al. The effect of pathophysiology on the surgical treatment of ischemic mitral regurgitation: operative and late risks of repair versus replacement. Eur J Cardiothorac Surg. 1995;9:568-74.

5. McGee EC, Gillinov AM, Blackstone EH, et al. Recurrent mitral regurgitation after annuloplasty for functional ischemic mitral regurgitation. J Thorac Cardiovasc Surg. 2004;128:916-24.

6. Grossi EA, Goldberg JD, LaPietra A, et al. Ischemic mitral valve reconstruction and replacement: comparison of long-term survival and complications. J Thorac Cardiovasc Surg. 2001;122:1107-24.

7. Seipelt RG, Schoendube FA, Vazquez-Jimenez JF, Doerge H, Voss M, Messmer BJ. Combined mitral valve and coronary artery surgery: ischemic versus non-ischemic mitral valve disease. Eur J Cardiothorac Surg. 2001;20:270-5.

8. Grigioni F, Enriquez-Sarano M, Zehr KJ, Bailey KR, Tajik AJ. Ischemic mitral regurgitation: long-term outcome and prognostic implications with quantitative Doppler assessment. Circulation. 2001;103: 1759-64.

9. Czer LS, Maurer G, Bolger AF, DeRobertis M, Chaux A, Matloff JM. Revascularization alone or combined with suture annuloplasty for ischemic mitral regurgitation. Evaluation by color Doppler echocardiography. Tex Heart Inst J. 1996;23:270-8.

10. Aklog L, Filsoufi F, Flores KQ, et al. Does coronary artery bypass grafting alone correct moderate ischemic mitral regurgitation? Circulation. 2001;104:I68-75.

11. Prifti E, Bonacchi M, Frati G, Giunti G, Babatasi G, Sani G. Ischemic mitral valve regurgitation grade II-III: correction in patients with impaired left ventricular function undergoing simultaneous coronary revascularization. J Heart Valve Dis. 2001;10:754-62.

12. Grossi EA, Bizekis CS, LaPietra A, et al. Late results of isolated mitral annuloplasty for "functional" ischemic mitral insufficiency. J Card Surg. 2001;16:328-32.

13. Hausmann $\mathrm{H}$, Siniawski $\mathrm{H}$, Hotz $\mathrm{H}$, et al. Mitral valve reconstruction and mitral valve replacement for ischemic mitral insufficiency. J Card Surg. 1997;12:8-14.
14. Flameng W, Herijgers P, Bogaerts K. Recurrence of mitral valve regurgitation after mitral valve repair in degenerative valve disease. Circulation. 2003;107:1609-13.

15. Bax JJ, Braun J, Somer ST, et al. Restrictive annuloplasty and coronary revascularization in ischemic mitral regurgitation results in reverse left ventricular remodeling. Circulation. 2004;110:II103-8.

16. Tahta SA, Oury JH, Maxwell JM, Hiro SP, Duran CM. Outcome after mitral valve repair for functional ischemic mitral regurgitation. J Heart Valve Dis. 2002;11:11-8; discussion 18-9.

17. Zoghbi W, Enriquez-Sarano M, Foster E, et al. Recommendations for evaluation of the severity of native valvular regurgitation with twodimensional and Doppler echocardiography. J Am Soc Echocardiogr. 2003; 16:777-802.

18. Kircher B, Abbott JA, Pau S, et al. Left atrial volume determination by biplane two-dimensional echocardiography: validation by cine computed tomography. Am Heart J. 1991;121:864-71.

19. D'Cruz I, Shroff S, Janicki J, Jain A, Reddy H, Lakier J. Differences in the shape of the normal, cardiomyopathic, and volume overloaded human left ventricle. J Am Soc Echocardiogr. 1989;2:408-14.

20. Kono T, Sabbah HN, Rosman H, Alam M, Jafri S, Goldstein S. Left ventricular shape is the primary determinant of functional mitral regurgitation in heart failure. J Am Coll Cardiol. 1992;20:1594-8.

21. Kono T, Sabbah HN, Stein PD, Brymer JF, Khaja F. Left ventricular shape as a determinant of functional mitral regurgitation in patients with severe heart failure secondary to either coronary artery disease or idiopathic dilated cardiomyopathy. Am J Cardiol. 1991;68:355-9.

22. Kumanohoso T, Otsuji Y, Yoshifuku S, et al. Mechanism of higher incidence of ischemic mitral regurgitation in patients with inferior myocardial infarction: quantitative analysis of left ventricular and mitral valve geometry in 103 patients with prior myocardial infarction. J Thorac Cardiovasc Surg. 2003;125:135-43.

23. Czer LS, Maurer G, Trento A, et al. Comparative efficacy of ring and suture annuloplasty for ischemic mitral regurgitation. Circulation. 1992;86:II46-52.

24. von Oppell UO, Stemmet F, Brink J, Commerford PJ, Heijke SA. Ischemic mitral valve repair surgery. J Heart Valve Dis. 2000;9:64-73; discussion 73-4.

25. Gorman RC, McCaughan JS, Ratcliffe MB, et al. Pathogenesis of acute ischemic mitral regurgitation in three dimensions. $J$ Thorac Cardiovasc Surg. 1995;109:684-93.

26. Levine RA, Messas E, Nathan NS, Rudski LG. New understanding of ischemic mitral regurgitation: the marionette and its masters. Eur $J$ Echocardiogr. 2004;5:313-7.

27. Kaul S, Spotnitz WD, Glasheen WP, Touchstone DA. Mechanism of ischemic mitral regurgitation. An experimental evaluation. Circulation. 1991;84:2167-80.

28. Otsuji Y, Handschumacher MD, Schwammenthal E, et al. Insights from three-dimensional echocardiography into the mechanism of functional mitral regurgitation: direct in vivo demonstration of altered leaflet tethering geometry. Circulation. 1997;96:1999-2008.

29. Yiu SF, Enriquez-Sarano M, Tribouilloy C, Seward JB, Tajik AJ. Determinants of the degree of functional mitral regurgitation in patients with systolic left ventricular dysfunction: a quantitative clinical study. Circulation. 2000;102:1400-6.

30. Agricola E, Oppizzi M, Maisano F, et al. Echocardiographic classification of chronic ischemic mitral regurgitation caused by restricted motion according to tethering pattern. Eur J Echocardiogr. 2004;5: 326-34.

\section{Discussion}

Dr Robert Klautz (Leiden, The Netherlands). Dr Bouchard and his group have presented their results on the surgical treatment of ischemic MR and have found a rather disappointing result: more than $57 \%$ of patients had recurrent MR of grade 2 or more at mid-term follow-up. This is in strong contrast to our own experience from Leiden published in Circulation last year, in which only 1 out of 51 patients had a recurrence of MR grade 2 and no patient had a grade 3 or 4 at 2 years' follow-up. In trying to reconcile these 
findings, I have tried to find explanations, and I would like your opinion on these thoughts.

First, there could be a difference in patient selection. Although in our study, ejection fraction was somewhat lower than in yours$31 \%$-you have included patients with more than just pure ischemic MR. Five patients had either aortic valve surgery or LV aneurysm resection. I think those should be excluded. Otherwise I cannot find a difference in our patients, and I cannot find an explanation for your worse outcomes on the basis of a worse patient population. If anything, our patients were a little sicker, NYHA class was lower, on the average, and LV dimensions were slightly bigger. So this does not seem the cause of the difference.

Second, and now I come to a more fundamental point, we found evidence of a time-dependent reverse remodeling. About one third of the patients showed reverse remodeling already at 3 months and another third at 18 months, and the final one third didn't show reverse remodeling. In your presentation, you did not mention the change in LV dimensions, but from your manuscript I understand that there was no change. Our results with a lack of recurrent MR could, of course, be explained by the fact that the ventricles of our patients got smaller, but I would hypothesize the reverse. They became smaller because there was no recurrent MR, and you did not find reverse remodeling because you had recurrence of MR.

Now I come to my questions. Did you indeed find no signs of reverse remodeling, and do you agree that the high recurrence rate of MR in your study might be the cause of that, rather than the consequence?

Dr Bouchard. Thank you for those very good comments. I have been looking very carefully at your data and also tried to reconcile the different results. In terms of patient selection, I think that we are dealing with slightly different patient populations, as you mentioned. Yours had a significantly lower ejection fraction to start with and larger ventricles. Our cohort had 29\% of acute myocardial infarction with $19 \%$ of reoperation and $8 \%$ of people having emergency surgery. I think that all those factors could distinguish the populations.

In terms of the Alfieri and the AVR, the results would be similar if we excluded those 2 subgroups. The AVR patients have been doing very well, with $1+$ MR on follow-up. As for the Alfieri patients, they are doing well clinically. They both had $2+\mathrm{MR}$ at follow-up.

In terms of reverse remodeling, that was a very interesting aspect. We did not experience reverse remodeling in our series. The ventricular dimensions and the ejection fractions were all similar from the preop stage to the delayed postop echo follow-up, and no reverse remodeling was found whether or not the patient had recurrent mitral regurgitation.

Now, in terms of having more recurrence, one hypothesis could be as follows: if the ventricles in our series are not as dilated as they are in your series, combined with the fact that many of our patients had acute myocardial infarction, that could leave us with ventricles with more room for negative remodeling or dilation during follow-up. That is just a hypothesis, because the patients with acute myocardial infarctions didn't behave differently from the rest of the cohort in terms of MR recurrence in our series.

Dr Klautz. Now, if the recurrence of MR is the cause of lack of reverse remodeling, then maybe we should look more carefully at your early results. You call them good, but immediately postoperatively you already had a $10 \% 2+$ MR rate, which, in our opinion, is not a good result. Our criteria for achieving a good repair in these patients with a restrictive type of MR is absolutely no residual MR in the OR [operating room] and a coaptation length of at least $8 \mathrm{~mm}$. If this is not achieved at the end of surgery, we consider replacing the ring and downsizing even further. By holding on to these principles of stringent downsizing, at discharge no patient had more than trivial MR.

Our median ring size was 26 , all complete rings, while from your slides, your median ring size was 28 , with some noncomplete rings, which brings me to my next question. Did you check the coaptation length at the end of surgery, and might it be possible that you did not achieve adequate treatment of the restriction by not downsizing despite the mere absence of MR at the end of surgery?

Dr Bouchard. These are good points. As for the $2+$ MR at the early postop echoes, those patients didn't behave statistically differently from the patients with better perioperative echo results in terms of MR recurrence at late follow-up. Obviously it is a small subgroup of patients, 7 patients. They tended, however, not to do as well clinically. So I would support being very aggressive and not leaving those $2+$ behind. Among those 7 patients, 2 died in the first 3 months, and 2 had mitral valve replacement during the follow-up period.

As for coaptation surface, we didn't specifically look at that. We did look, however, at whether there was a difference or not in terms of using a complete ring or a posterior band, and those results were similar in terms of recurrence.

Dr Klautz. Finally, I want to come back to the patients in our series who did not show reverse remodeling, about one third of the patient group. These were the patients with the bigger preoperative end-diastolic dimension, and this was the most important predictor of reverse remodeling. We could not analyze predictors of recurrent MR because there were none. When analyzing our results with ROC [receiver operating characteristic] curves, we found that a preop end-diastolic dimension of 65 and end-systolic dimension of 51 are the optimal cutoff values below which reverse remodeling is predictable. We therefore believe that when the heart is larger than that, additional procedures should be employed. In Leiden, we think that for patients with end-diastolic dimensions between 65 and $80 \mathrm{~mm}$, additional CorCap placement could be a valuable adjunct. What are your thoughts on this subject?

Dr Bouchard. Well, we didn't have any experience with chordal shortening. It is certainly an interesting approach considering that leaflet tethering is at the center of this pathology. All our patients had some form of leaflet tethering, and we happened in our echo subgroups to find that the shorter tethering distance between the posterior papillary muscle and the anterior annulus had some effect. I would assume that some form of treatment at the level of the chordae could have an effect. We just didn't have firsthand experience with those techniques.

Dr Klautz. I am sorry, I wasn't talking about a chordal procedure but an additional procedure on the ventricle itself, like a CorCap or a cardiac support device.

Dr Bouchard. We did not have experience with that ventricular approach either. 
Dr Christopher Acar (Paris, France). Dr Bouchard, I enjoyed your presentation. I think it would be dangerous to leave the room keeping in mind that intraoperative echo is not a useful adjunct for mitral valve repair even though there are limitations in ischemic mitral valve insufficiency. In fact we don't have any other reliable test available in the operating room. Did you consider increasing the sensitivity of the intraoperative echo by raising the systemic pressure with vasoconstrictors?

Dr Bouchard. Well, we had some experience with that, like most centers, except that during the time span of this study, that was not done in any routine or systematic fashion. But especially with ischemic MR or functional MR, we tried to challenge those patients. We tried to challenge those patients with a decent afterload, probably a mean pressure in the 70 to 80 range, and also with volume challenge to try to give them a filling pressure that is similar to what they had before their surgery.

These are very good points. In this study, we didn't look specifically at whether challenging those patients in the OR would give our early postoperative results a more meaningful predictor of MR recurrence. However, a very large majority of those patients had zero regurgitation in the OR setting, which might make them less likely to have significant MR when we challenged them.

Dr Acar. There was no residual MR even with an increased afterload?

Dr Bouchard. When that was done, which was not standardized during that period of the study.

Dr Khalid Rasheed (Islamabad, Pakistan). I noticed that only $88 \%$ of your patients had associated coronary revascularization. I believe that for an ischemic MR repair to succeed, it is imperative that all patients be revascularized. Why did $12 \%$ not have any revascularization, and could this be a factor in a higher recurrence of mitral regurgitation in your series?

Dr Bouchard. This is a very good question. Some patients were already revascularized - they were reoperations - and a large proportion of those patients had all their grafts patent. We did, however, look specifically at incomplete revascularization and whether or not that would be part of why some of the patients had recurrent MR, and that didn't seem to be associated with recurrence of MR.

Dr David Adams (New York, NY). I would just like to make 2 comments for your consideration. The first is about your grading of MR. You have a very qualitative scale. Can you tell us a little more about how these were graded? Were they blinded? Were they double reviewed? How did you actually look at your postop MR grade?

Dr Bouchard. Most of the echoes, that means all the echoes except 9 , were done in our institution. The grading was dependent on the specific echocardiographer reviewing the exam, but as an institution, they tend to grade the mitral regurgitation in a similar fashion. So the grade 1 MRs were those where the MR jet area compared to the left atrial area was below 20\%; the 2+ MRs were those where the jet area was between $20 \%$ and $40 \%$; the $3+$ MRs were those where the jet area was above $30 \%$ and had systolic pulmonary reflux; and the grade 4 would be kept for those with more than $40 \%$ of the MR jet area as well as systolic pulmonary vein reversal. That is a pretty strict and severe way of looking at MR. In the 18 patients of the echo subgroups, the tapes were looked at by only 1 echocardiographer, and these were the same criteria used during these evaluations.
Dr Adams. I would recommend that you go back and have the same echocardiographer review all of your echoes. I know several of us have done this internally, and it is surprising what happens when you have 1 echocardiographer in your institution go back and relook at your official echo reports when you are using semiquantitative or qualitative echo grades.

The second question I have is specifically about sizing, because your results are different than several experiences, not so much in terms of recurrent MR, because very little has been published about that, but in terms of residual MR with a downsized remodeling ring, that in fact has been shown in several series to be very low, and can you tell us again exactly how you size these rings, and how did you decide the $80 \%$ that got a remodeling ring versus the $20 \%$ that didn't?

Dr Bouchard. These are good questions. First, in answer to having all the echoes reviewed by the same person, that is what was aimed at, and only a small part of the echoes were actually physically available for review. So the rest had to go with what reports were available.

In terms of ring sizing, those surgeries were done by a variety of our staff surgeons, some preferring at the time a posterior band, some preferring a Carpentier Physio ring. I cannot speak for all the surgeons, but very often the ring sizing was aimed at reducing by 2 sizes and ended up being size 26 for women and size 28 for men. As a group, we moved mainly to use a complete ring and moved to use either the Carbomedics Annuloflo or the Carpentier-McCarthy-Adams rings, which have, as you well know, a shorter septolateral distance, and I think that is an important issue.

Dr Adams. I would just again reiterate what you said, that you need to downsize these rings, and you need to go back as best you can, if you can, and tell us what percent of these rings were truly downsized, because the concept of just saying I am going to put a 28 in men and a 26 in women, that may not be downsizing. As you know, a lot of men may need a 24 Physio ring or a 26; this new ring that is downsized really is a dimension of 24 in the P3 dimension. So I would not say that just saying we are using a 28 in men really correlates to downsizing.

Dr Harold Roberts (Lauderdale Lakes, Fla). I enjoyed your presentation and your unflinching honesty. I do think that your lessthan-stellar results are partially self-inflicted, however. I believe $12 \%$ of your patients had an incomplete ring implanted. I personally have reoperated on 4 patients initially done by other surgeons that have had repairs for ischemic MR with Cosgrove bands that looked great when they went out of the OR and then a few months later they were leaking substantially. It is now well known that dilatation of the anterior annulus as well as reduction of the septal-lateral dimension are important concepts best accomplished by a complete, probably semirigid ring like the ET Logix or Physio.

The other thing that nobody really mentioned but that I think is helpful so that you can have zero or trace MR coming out of the OR is to close the naturally occurring scallops between P1 and P2 as well as between P2 and P3. This can be done with 2 rows of continuous 5-0 Cardionyl. Though it takes a few extra minutes, it is the often difference between trace and zero mitral regurgitation at the end of the procedure. Zero regurgitation should be the goal so that a margin of safety is present should there be any deterioration of the repair with time. 
Apical 4-chamber

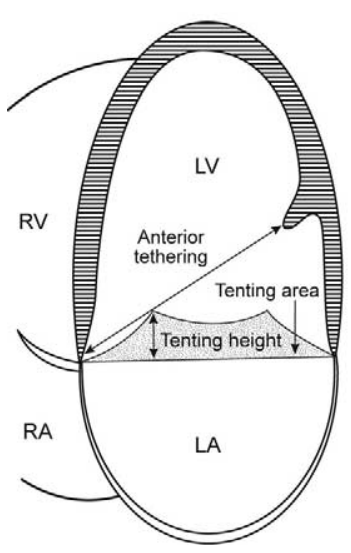

Apical 2-chamber

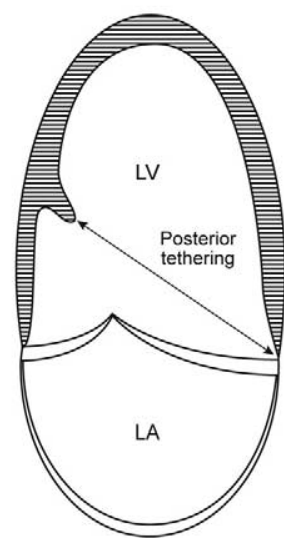

Figure E1. Echocardiographic measurements. $R V$, Right ventricle; $L V$, left ventricle; $R A$, right atrium; $L A$, left atrium.

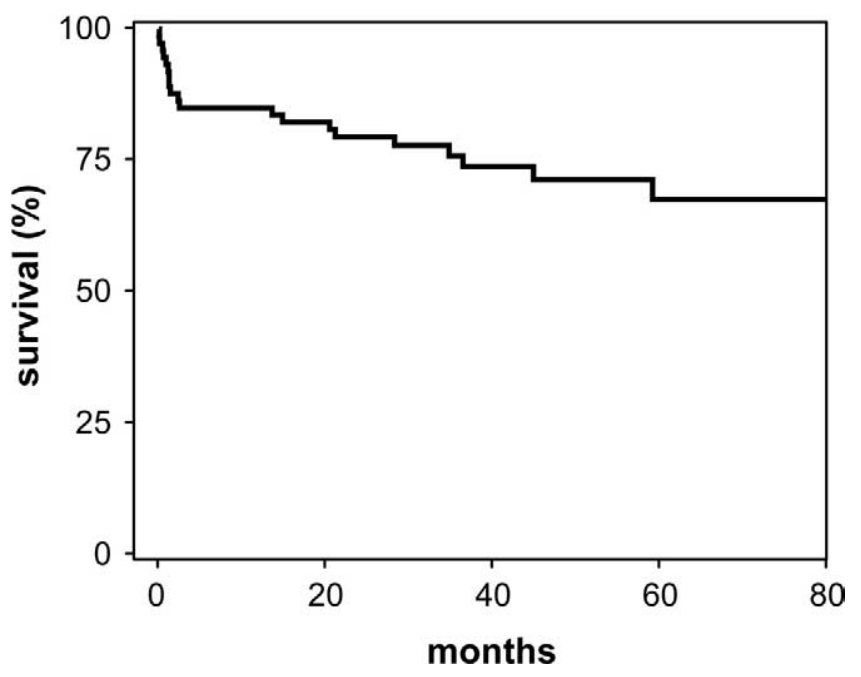

Figure E2. Kaplan-Meier survival for the overall study group. 


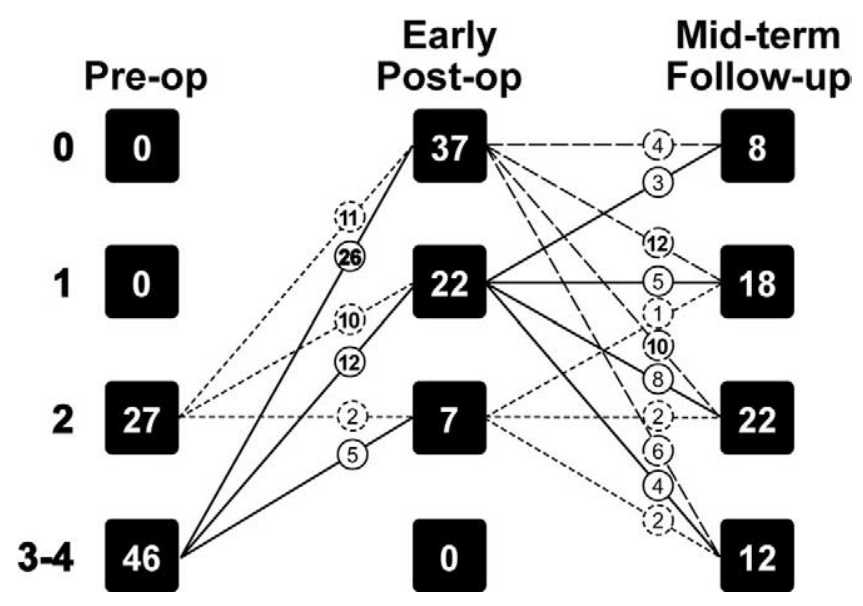

Figure E3. Changes in MR grade. 